\title{
The effect of oral contraceptives on aromatase and Cox-2 expression in the endometrium of patients with idiopathic menorrhagia or adenomyosis
}

\author{
This article was published in the following Dove Press journal: \\ International Journal of Women's Health \\ 12 June 2013 \\ Number of times this article has been viewed
}

\author{
Hugo Maia $\mathrm{Jr}^{1-3}$ \\ Clarice Haddad ${ }^{3}$ \\ Nathaniel Pinheiro ${ }^{4}$ \\ Julio Casoy ${ }^{3}$ \\ 'School of Medicine, Federal \\ University of Bahia, Salvador, Bahia, \\ Brazil; '2ltaigara Memorial Day \\ Hospital, Salvador, Bahia, Brazil; \\ ${ }^{3}$ CEPARH (Centro de Pesquisas \\ e Assistência em Reprodução \\ Humana), Salvador, Bahia, Brazil; \\ ${ }^{4}$ Imagepat Pathology Laboratory, \\ Federal University of Bahia, Salvador, \\ Bahia, Brazil
}

\begin{abstract}
Background: The presence of aromatase and cyclooxygenase-2 (Cox-2) expression was investigated in the endometrium of patients with idiopathic menorrhagia or adenomyosis. The effect of oral contraceptives administered in extended regimens on the endometrial expression of these enzymes was also investigated.

Methods and results: Aromatase expression was detected by immunohistochemistry in the endometrial glands and stroma of patients with idiopathic menorrhagia or adenomyosis. There was no difference in the percentage of aromatase expression in the endometria between the two groups. The mean intensity of Cox-2 expression in the glandular epithelium also did not differ significantly between the groups. Among the patients using oral contraceptives in extended regimens, the relative decrease in both aromatase and Cox-2 expression was significantly greater in amenorrheic patients compared with those who were experiencing breakthrough bleeding.

Conclusion: The presence of aromatase expression in the endometrium is associated with the occurrence of menorrhagia, irrespective of the presence of adenomyosis. Continuous expression of these enzymes in the endometrium of users of oral contraceptives in extended regimens is positively associated with the presence of breakthrough bleeding. This suggests a role for both aromatase and Cox-2 in the etiology of abnormal uterine bleeding.
\end{abstract}

Keywords: menorrhagia, aromatase, endometrium, Cox-2, adenomyosis

\section{Introduction}

Idiopathic menorrhagia has been defined as menstrual loss exceeding $80 \mathrm{~mL}$ of blood, in a woman with a normal uterus. The cause of menorrhagia is functional and is related to increased prostaglandin production as a consequence of the augmented cyclooxygenase-2 (Cox-2) levels in the endometrium resulting from increased messenger ribonucleic acid (mRNA) production. ${ }^{1}$ Although nonsteroidal antiinflammatory drugs (NSAID) are effective in reducing the amount of blood loss, other forms of treatment, such as oral contraceptives, levonorgestrel intrauterine systems, and endometrial ablation have also been shown to be effective. ${ }^{2-4}$

Progestins are capable of inhibiting Cox-2 expression in endometrial cells, and this may be one of the mechanisms responsible for the efficacy of oral contraceptives in the treatment of abnormal uterine bleeding and dysmenorrhea. ${ }^{5,6}$ It has yet to be established whether the increased prostaglandin production in the endometrium of patients with menorrhagia, which may be the underlying cause of this condition,
Correspondence: Hugo Maia Jr CEPARH, Rua Caetano Moura, 35, 402 10-34I Salvador, Bahia, Brazil Tel +55 7l 32478216

Fax +55 7I 32343442

Email ceparh@uol.com.br 
results from augmented estrogenic action in this tissue. Estrogens are known to upregulate Cox-2 expression in endometrial stromal cells, thus creating a positive feedback loop between estrogens and prostaglandins, which will have an enhancing effect on endometrial bleeding following hormone withdrawal. ${ }^{7}$ As occurs in endometriosis, this increase in estrogen production may not originate in the ovary but rather in the endometrium itself, through the action of locally expressed aromatase enzymes capable of converting testosterone and other androgens into estrogens. ${ }^{8}$ In a study of patients with severe dysmenorrhea and a normal pelvis at laparoscopy, aromatase expression was already detectable in the endometrium, thus suggesting that the presence of this enzyme may exacerbate menstrual pain, even in the absence of endometriosis. ${ }^{9}$ Since dysmenorrhea and excessive menstrual bleeding are commonly present together and both symptoms are exacerbated whenever Cox-2 expression increases in the endometrium, it is equally possible that local endometrial aromatase expression may also be the underlying cause of idiopathic menorrhagia occurring in patients with a normal uterus and ovulatory cycles. ${ }^{10}$ In estrogen-dependent pathologies, such as adenomyosis, myomas, or endometriosis, aromatase expression can be detected in the endometrium, although it is still to be confirmed whether similar enzymatic changes are already detectable in patients with idiopathic menorrhagia. ${ }^{11-14}$

One reasonable clinical conjecture is that the functional changes in the endometrium that lead to an increase in local estrogen production may not only be the underlying cause of idiopathic menorrhagia and primary dysmenorrhea, but may also favor the subsequent development of uterine pathologies associated with these symptoms. Studies carried out in primates have shown that chronic exposure of the endometrium to locally administered estrogens leads to the development of pathologies such as adenomyosis, polyps, and endometrial hyperplasia, thus mimicking the conditions encountered in the uterus when aromatase is expressed in the endometrium of these patients. ${ }^{15}$

In the present paper, the presence of aromatase and Cox-2 expression was investigated in the endometrium of patients with a history of menorrhagia who submitted to endometrial resection and whose pathology report showed either a normal uterus or adenomyosis. The effect of preoperative treatment with oral contraceptives on the endometrial expression of theses enzymes was also investigated, since these contraceptive pills are often prescribed to patients with menorrhagia, with the objective of reducing bleeding and thinning the endometrium prior to endometrial resection.

\section{Material and methods}

This was an observational study, the objective of which was to determine the presence of aromatase and Cox-2 expression in endometrial tissue samples obtained from patients with symptoms of excessive menstrual bleeding and dysmenorrhea. The study population consisted of 128 consecutive patients referred to this center at the Itaigara Memorial Day Hospital, Salvador, Bahia, Brazil for endometrial resection (using a bipolar electrode [Gynecare Versapoint ${ }^{\circledR}$, Ethicon, Cincinnati, OH, USA]) between January 2011 and February 2012 and who fulfilled the pathologic diagnostic criteria for either adenomyosis or a normal uterus. In brief, the surgical technique consisted of dilating the cervix to $9 \mathrm{~mm}$ to allow the introduction of a bipolar resectoscope (Gynecare Versapoint). A $0.9 \%$ isotonic saline solution was used to distend the uterine cavity. The endometrium was removed during surgery, together with approximately $5 \mathrm{~mm}$ of the underlying myometrium. This procedure was performed in a private hospital, and the patients were discharged 6 hours after its completion. In all the patients, the hysteroscopic procedure was carried out under intravenous sedation with propofol, in combination with a paracervical block established with an injection of $5 \mathrm{~mL}$ of $1 \%$ lidocaine at the cervicovaginal junction at the 4 and 8 o'clock positions.

All the surgical procedures were performed by the same group of surgeons ( $\mathrm{HM}$ and $\mathrm{CH}$ ). The histological and immunohistochemical analysis of the endometrium and myometrium were performed by the same pathologist (NP) in all cases.

Data on the menstrual-related symptoms, such as menorrhagia, dysmenorrhea, and the premenstrual syndrome reported by these patients prior to treatment were obtained from their medical records. A total of 39 patients in this study reported use of oral contraceptives in continuous regimens at the time of surgery. Information on the type of contraceptive used, the duration of treatment, and whether breakthrough bleeding and pain were present was obtained at interviews with the patients at the time of admission to hospital. The oral contraceptives were prescribed by the patients' attending physician to treat menorrhagia prior to their referral to this institute. The patients were using oral contraceptive formulations containing either $30 \mathrm{mcg}$ of ethinyl estradiol with 75 mcg of gestodene (Gestinol, Libbs Farmaceutica Ltda., São Paulo, Brazil) $(\mathrm{n}=23)$ or $30 \mathrm{mcg}$ of ethinyl estradiol with $3 \mathrm{mg}$ of drospirenone (Libbs Farmacêutica) $(\mathrm{n}=16)$. Since both formulations had already been approved by the regulatory authorities in Brazil (Agência Nacional de Vigilância Sanitária [ANVISA]) for continuous use to suppress 
menstruation, in this study, the oral contraceptives were used in extended regimens. No patients in the study population reported currently using Cox-2 inhibitors or other steroids at the time of admission to hospital for hysteroscopy.

During hysteroscopy performed using the Versapoint resectoscope, the endometrium and approximately $0.5 \mathrm{~cm}$ of the myometrium were removed as described above, fixed in $4 \%$ formalin, and sent to pathology for histological and immunohistochemical evaluation. Immunohistochemistry was performed following antigen retrieval to detect the presence of aromatase P450 and Cox-2 expression. Aromatase expression was investigated using a commercially available monoclonal mouse anti-human cytochrome $\mathrm{P} 450$ aromatase antibody, clone H4 (Dako, Carpinteria, CA, USA), while Cox-2 expression was assessed using monoclonal mouse anti-human Cox-2 antibody (CX-294). Antigen retrieval was performed using a tris (hydroxymethyl)aminomethane (Tris) -ethylenediaminetetraacetic acid (EDTA) (Sigma Aldrich, St. Louis, MI, USA) buffer at $\mathrm{pH}$ 8.0. The reaction was revealed using the streptavidin-biotin method. The presence of aromatase expression was rated either as positive if there was any detectable staining reaction in the glandular epithelium or negative when no reaction was observed. Cox-2 expression was graded as 0 when no expression was detected, +1 when there was a light staining in more than $10 \%$ of the glands, +2 when staining was moderate, and +3 when there was strong expression in the endometrium. The Cox- 2 reaction is labeled strong when the intensity is the same as that found in the leukocytes present in the tissue specimen. The slides were evaluated by the same pathologist in all cases. One tissue section was taken from each endometrial sample for immunohistochemical analysis. Placental tissue and samples of the atrophic endometrium obtained from the pathology archives were used as positive and negative controls, respectively, in all immunostaining reactions for aromatase P450.

Statistical analysis was performed using the chi-square test to detect differences in percentages and Student's $t$-test for comparison of means. Significance was established at $P<0.05$.

All patients gave their written informed consent authorizing the immunohistochemical studies to be performed on the endometrial tissue, as approved by the internal review board of the Itaigara Memorial Day Hospital. All the surgical procedures were part of the standard medical care for the treatment of menorrhagia at the Itaigara Memorial Day Hospital, and no manipulation was done to allocate these patients for surgery on specific days of the menstrual cycle because they were involved in a research study.
The criteria used to diagnose idiopathic menorrhagia was the self-reported occurrence of heavy uterine bleeding with the passage of blood clots, absence of abnormal uterine or adnexal findings at transvaginal ultrasonography, a pathology-free uterine cavity at hysteroscopy, and a pathology report showing no histological abnormalities. The patients enrolled to the study were divided into three groups, in accordance with the International Federation of Gynecology and Obstetrics (FIGO) classification (the PALM-COEIN classification $)^{25}$ of abnormal uterine bleeding: group A, which consisted of 45 patients with a mean body mass index (BMI) of $27 \pm 5 \mathrm{~kg} / \mathrm{m}^{2}$ (mean \pm standard deviation [SD]) whose postoperative pathology report showed a histologically normal endometrium and myometrium; group B, which consisted of 44 patients with a BMI of $29 \pm 7 \mathrm{~kg} / \mathrm{m}^{2}$ and a histologically proven diagnosis of adenomyosis; and group $\mathrm{C}$, which consisted of 39 patients with a BMI of $25 \pm 6 \mathrm{~kg} / \mathrm{m}^{2}$ and a history of menorrhagia who were using oral contraceptives in extended regimens prior to the hospital admission. In this last group, the patients were divided into two subsets, based on whether or not they had reported episodes of breakthrough bleeding at the time of surgery. This oral contraceptive group (C) was further classified by the pathologist as having either adenomyosis $(n=13)$ or a normal uterus $(n=26)$.

\section{Results}

In the patients of group A, the endometrium was found to be in the proliferative phase in 34 cases, while in the remaining eleven cases, secretory changes were found. The underlying myometrium was normal, and the uterine volume, as determined by transvaginal ultrasonography, was in the normal range $\left(100 \pm 20 \mathrm{~cm}^{3}\right)$.

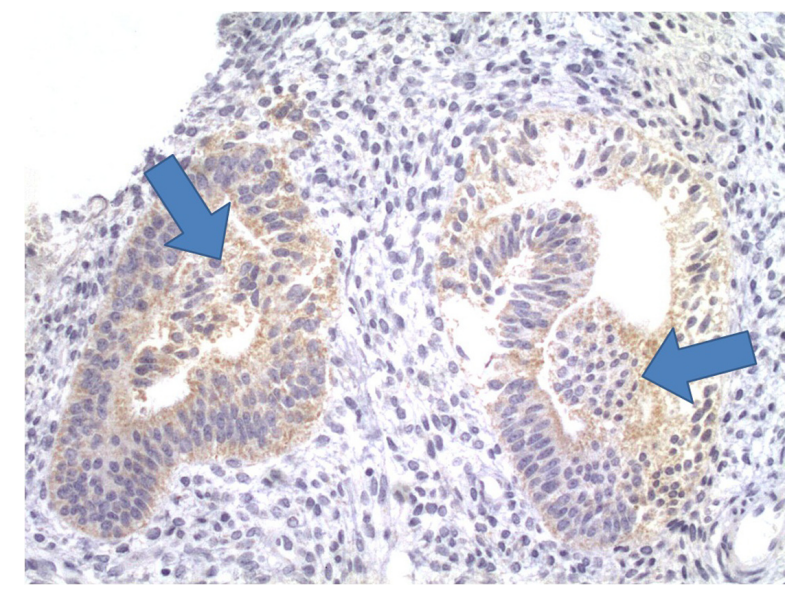

Figure I Aromatase expression in the glandular epithelium of the endometrium, in a patient with a normal uterus and symptoms of menorrhagia. Arrows point to areas of aromatase expression in the glandular epithelium. 
Aromatase expression was present in the endometrial glands or stroma in $80 \%$ of the patients with idiopathic menorrhagia (Figure 1). In patients with adenomyosis and symptoms of menorrhagia, P450 aromatase was detected by immunohistochemistry in both the eutopic endometrium and in the adenomyosis lesions, in $74 \%$ of the cases. In this group, 27 patients were in the proliferative phase, while the remaining 17 were in the luteal phase. There were no statistically significant differences in aromatase expression between groups A (endometrial) and B (adenomyosis).

Likewise, there was no statistically significant difference in the mean score for Cox-2 expression in the glandular epithelium, between patients with idiopathic menorrhagia and those with adenomyosis (Figure 2). These results are summarized in Table 1.

\section{Aromatase expression in oral contraceptive users}

In patients who were using oral contraceptives at the time of endometrial resection (group C), the percentage of endometrial tissue showing a positive reaction for aromatase in either the stromal or glandular epithelium was significantly less than that found in the nonusers (groups A + B). However, when the oral contraceptive users were separated into two subsets according to the presence or absence of amenorrhea at the time of hospital admission, the cases that were found to be positive for aromatase expression were all in the group with breakthrough bleeding and pain. In those patients who became amenorrheic during oral contraceptive use, aromatase

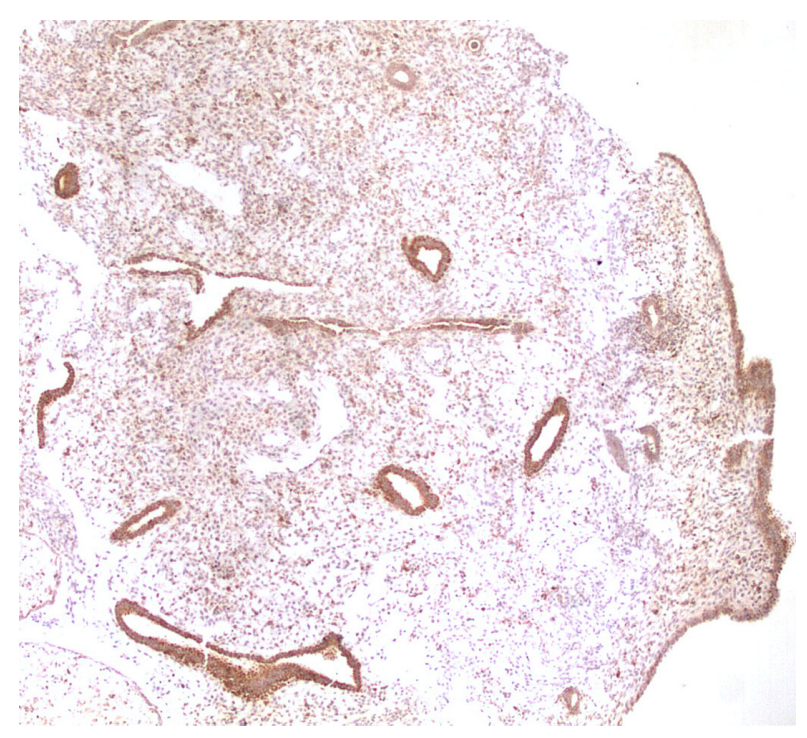

Figure 2 Cox-2 expression in the endometrial glands of a patient with idiopathic menorrhagia.

Abbreviation: Cox-2, cyclooxygenase-2.
Table I Aromatase and Cox-2 expression in the endometrium of patients with idiopathic menorrhagia or adenomyosis

\begin{tabular}{lll}
\hline & $\begin{array}{l}\text { Aromatase } \\
\text { (\% positive) }\end{array}$ & $\begin{array}{l}\text { Cox-2 } \\
\text { (mean score) }\end{array}$ \\
\hline Idiopathic menorrhagia & $36 / 45(80 \%)$ & 1.7 \\
Adenomyosis & $32 / 43(74 \%)^{*}$ & $1.6^{* *}$ \\
\hline
\end{tabular}

Notes: $* P=0.7(\mathrm{NS}) ; * * P=0.18(\mathrm{NS})$.

Abbreviations: Cox-2, cyclooxygenase-2; NS, not significant.

expression was not detected in any of the endometrial samples, either from normal or adenomyosis-bearing uteri $(n=27)$ (Figure 3). These results are summarized in Table 2. However, the incidence of breakthrough bleeding and amenorrhea in this study population does not reflect the actual incidence in the general population of oral contraceptive users because the selection process for surgery may have introduced a bias.

There was no correlation between the occurrence of breakthrough bleeding and the type of progestin present in the extended oral contraceptive pill regimens, which in most cases consisted either of gestodene or drospirenone. The duration of contraceptive use prior to endometrial resection, a mean of 5 months in both the amenorrhea and breakthrough bleeding groups, had no effect on the incidence of breakthrough bleeding. The mean scores for Cox-2 expression in the glandular epithelium were significantly lower in amenorrheic oral contraceptive users compared with those reporting breakthrough bleeding (0.6 versus 1.9). This difference was statistically significant $(P=0.03)$.

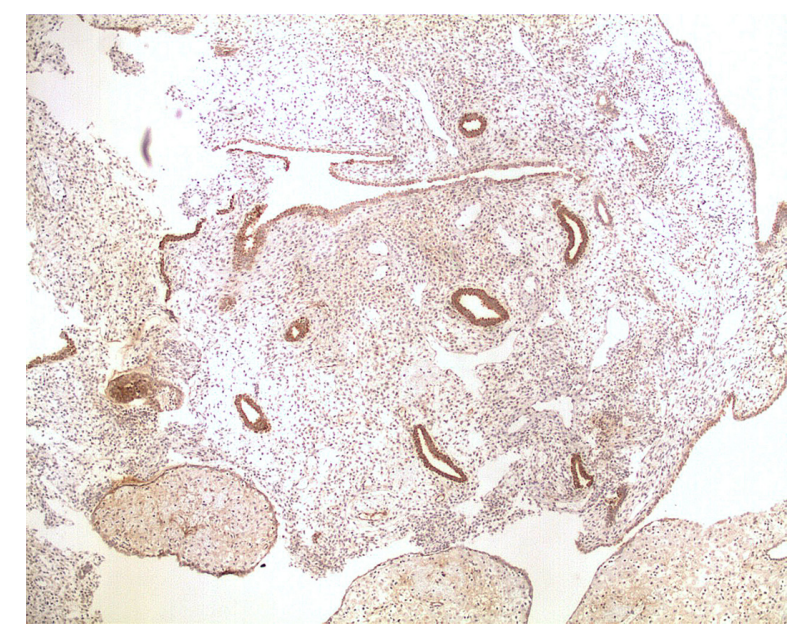

Figure 3 Aromatase expression in both the atrophic endometrial glands and decidual stroma, in a patient with idiopathic menorrhagia who was experiencing breakthrough bleeding during the use of an oral contraceptive containing drospirenone in an extended regimen. 
Table 2 Aromatase expression in oral contraceptive users in relation to the presence of breakthrough bleeding, when compared with nonusers

\begin{tabular}{ll}
\hline & $\begin{array}{l}\text { Positive aromatase } \\
\text { expression }\end{array}$ \\
\hline Nonusers (groups A + B) & $68 / 88(77 \%)$ \\
Oral contraceptive users (group C) & $7 / 39(18 \%)^{*}$ \\
Oral contraceptive users in amenorrhea & $0 / 27(0 \%)$ \\
Oral contraceptive users with & $7 / 12(60 \%)^{* * * * * *}$ \\
breakthrough bleeding & \\
\hline
\end{tabular}

Notes: *Significantly less than in nonusers $(P<0.001)$; **not significant when compared with nonusers. ***significantly different from the group of oral contraceptive users with amenorrhea $(P<0.01)$.

\section{Discussion}

The present results showed that aromatase expression was detectable in the endometrium of patients with menorrhagia even in the absence of any uterine pathology. It is important to mention that the percentage of endometrial samples that tested positive for aromatase expression did not differ significantly between patients with idiopathic menorrhagia and those with adenomyosis, thus suggesting that there may be no difference between these two groups with respect to the mechanisms behind this excessive uterine bleeding. This is in agreement with previous studies conducted with primates, showing that estradiol administered locally into the uterine cavity was able to provoke the development of various uterine pathologies, including adenomyosis. ${ }^{15}$ This suggests that the continuous exposure of the endometrium to locally produced estradiol may foster the development of estrogen-dependent pathologies. The persistent local exposure to estrogens in this experimental setting mimics the conditions found in the endometrium of patients in whom aromatase expression is positive. In these women, there is a hyperestrogenic milieu in the uterus that may exert an antagonistic effect on progesterone. ${ }^{16}$ This would initially exacerbate menstrual bleeding by upregulating Cox-2 expression, prior to promoting the development of adenomyosis. The present findings that the intensity of Cox-2 expression in the endometrium did not differ significantly between the normal uteri and those with adenomyosis further corroborate the conjecture that the mechanisms of excessive menstrual bleeding are similar in these two groups.

Idiopathic menorrhagia and adenomyosis may represent different facets of the same functional changes in the endometrium and likely constitute different steps in the development of this disease. Unfortunately, it is impossible to infer from this observational study whether aromatase expression predates the development of adenomyosis chronologically, since there are no data from epidemiological or clinical studies showing that patients with idiopathic menorrhagia progress to adenomyosis if left untreated. However, in patients with idiopathic menorrhagia submitted to endometrial resection who had to undergo a second procedure because of the recurrence of menstrual bleeding, the second pathology report showed a progression from a uterus that was originally pathology-free to one with adenomyosis. ${ }^{17}$ Although this is in agreement with experimental studies in primates showing that a local infusion of estradiol into the uterine cavity triggers the development of adenomyosis, it still remains to be established whether a similar situation occurs in women when the endometrium begins to express aromatase in a disease-free uterus. Clinical observations have shown that success rates vary little between patients with idiopathic menorrhagia and those with adenomyosis, when submitted to endometrial resection, thus suggesting that the mechanisms of action may be similar. The presence of adenomyosis does not seem to exert a negative impact on the results of the endometrial resection, therefore suggesting that at least in terms of clinical response, the presence of this pathology does not appear to affect results. ${ }^{18}$

The resumption of bleeding following endometrial resection is mainly attributed to the immense regenerative capacity of the endometrial cells, which is further enhanced by the increased local production of estrogens. In this respect, as has been suggested for endometriosis, the appearance of adenomyosis is a later consequence of this aberrant aromatase expression in the endometrium. ${ }^{19}$ The time sequence of enhanced aromatase expression in the endometrium occurring prior to the subsequent development of any pathology may explain the long delay between the onset of menstrual symptoms and the clinical diagnosis of estrogen-dependent pathologies. ${ }^{9}$ This is precisely what would be expected from our findings of similar enzymatic alterations in the endometria of patients with menorrhagia with or without adenomyosis, thus suggesting that an increase in local estrogen production is positively associated with the exacerbation of menstrual symptoms, such as bleeding and pain, and that these symptoms occur either independently or prior to the development of uterine pathology.

The association between persistent aromatase expression in the endometrium of patients reporting breakthrough bleeding during the continuous use of oral contraceptives is in agreement with the hypothesis that unabated aromatase expression is associated with persistent bleeding. In these patients, the progestins present in the oral contraceptive formulations may fail to inhibit aromatase gene transcription in the endometrium, thus triggering a vicious cycle of persistent 
local estrogen action and increased Cox-2 expression, and the ensuing production of proinflammatory prostaglandins, such as prostaglandin E2 (PGE2). This is in agreement with the findings reported here of increased Cox-2 expression in the endometrium of patients experiencing breakthrough bleeding during oral contraceptive use.

The presence of aromatase expression in the endometrium of endometriosis patients correlated positively with the severity of both endometriosis and adenomyosis, which are also commonly associated pathologies. The increased aromatase expression in the endometrium is due to an increase in the amount of mRNA transcripts for aromatase, thus suggesting that enhanced gene transcription may be the underlying mechanism that triggers this aggravation in menstrual-related symptoms and the development of this pathology. ${ }^{19}$

The persistent aromatase expression in the eutopic endometrium of menorrhagia patients with breakthrough bleeding during oral contraceptive use maintains Cox-2 and nuclear factor kappa-light-chain-enhancer of activated B cells (NF-kappa B) activity unabated, and this may be the triggering mechanism behind the persistence of breakthrough bleeding and dysmenorrhea reported by these patients. ${ }^{20}$ These findings are in agreement with the hypothesis that the aromatase expression in the endometrium of oral contraceptive users must be resolved in order to achieve a state of amenorrhea and to resolve menstrualrelated symptoms.

A capacity to block aromatase expression has also been reported with respect to other progestins, which appear to work at gene transcription level. ${ }^{14,21,22}$ This suggests that local estrogen production alone, irrespective of the presence of adenomyosis, is the underlying mechanism responsible for menorrhagia and dysmenorrhea and that this local hyperestrogenism is a consequence of an increased inflammatory milieu. ${ }^{23}$ From a biological point of view, this is a plausible possibility since increased local estrogen production in endometrial tissue will upregulate Cox-2 expression, thus leading to a greater production of proinflammatory prostaglandins, which in turn, will exacerbate menstrual bleeding and pain. ${ }^{6,23}$ This explains the present finding that failure to achieve amenorrhea with the continuous use of oral contraceptives to treat menorrhagia is a consequence of progesterone resistance caused by persistent aromatase and Cox- 2 expression in the endometrium. These findings also explain why the use of oral contraceptives in continuous regimens in conjunction with plant-derived aromatase inhibitors is associated with a lesser incidence of breakthrough bleeding and pain in patients with endometriosis compared with the use of oral contraceptives alone. ${ }^{24}$

In conclusion, the present findings suggest that local estrogen production in the endometrium may play a pivotal role in increasing menstrual flow, thus constituting the underlying mechanism responsible for the development of idiopathic menorrhagia.

Using the recent FIGO classification system for abnormal uterine bleeding (the PALM-COEIN classification), one may conclude from the present study that enzymatic changes in the endometrium were similar in patients allocated to the endometrial or adenomyosis groups, possibly indicating that there is a common underlying mechanism behind the abnormal uterine bleeding in these patients irrespective of the presence of pathology. ${ }^{25}$

\section{Disclosure}

The authors report no conflicts of interest in this work.

\section{References}

1. Smith OP, Jabour HN, Critchley HP. Cyclooxygenase enzyme expression and $\mathrm{E}$ series prostaglandin receptor signalling are enhanced in heavy menstruation. Hum Reprod. 2007;22(5):1450-1456.

2. Maia HJ, Casoy J. Non-contraceptive health benefits of oral contraceptives. Eur J Contracept Reprod Health Care. 2008;13(1): $17-24$.

3. Fraser IS, Kovacs GT. The efficacy of non-contraceptive uses for hormonal contraceptives. Med J Aust. 2003;178(12):621-623.

4. Maia H Jr, Maltez A, Coelho G, Athayde C, Coutinho EM. Insertion of Mirena after endometrial resection in patients with adenomyosis. $J \mathrm{Am}$ Assoc Gynecol Laparosc. 2003;10(4):512-516.

5. Maia H Jr, MaltezA, Studard E, Zausner B, Athayde C, Coutinho E. Effect of menstrual cycle and oral contraceptives on cycloooxygenase-2 expression in the endometrium. Gynecol Endocrinol. 2005;21(1):57-61.

6. Koike H, Egawa H, Ohtsuka T, Yamaguchi M, Ikenoue T, Mori N. Correlation between dysmenorrheic severity and prostaglandin production in women with endometriosis. Prostaglandins Leukot Essent Fatty Acids. 1992;46(2):133-137.

7. Noble LS, Takayama K, Zeitoun KM. Prostaglandin E2 stimulates aromatase expression in endometriosis-derived stromal cells. J Clin Endocrinol Metab. 1997;82(2):600-606.

8. Bulun S, Gurates B, Fang Z, et al. Mechanisms of excessive estrogen formation in endometriosis. $J$ Reprod Immunol. 2002;55(1):21-33.

9. Maia H Jr, Haddad C, Casoy J. Correlation between aromatase expression in the eutopic endometrium of symptomatic patients and the presence of endometriosis. Int J Women's Health. 2012;4:61-65.

10. Ferenczy A. Pathophysiology of adenomyosis. Hum Reprod Update. 1998;4(4):312-322.

11. Maia H Jr, Casoy J, Valente Filho J. Is aromatase expression in the endometrium the cause of endometriosis and its related infertility? Gynecol Endocrinol. 2010;26(4):253-257.

12. Maia H Jr, Pimentel K, Casoy J, et al. Aromatase expression in the eutopic endometrium of myomatous uteri: the influence of the menstrual cycle and oral contraceptive use. Gynecol Endocrinol. 2007;23(6): 320-324.

13. Maia H Jr, Casoy J, Correia T, Freitas A, Pimentel K, Coutinho EM. Effect of the menstrual cycle and oral contraceptives on aromatase and cycooxygenase-2 expression in adenomyosis. Gynecol Endocrinol. 2006;22(10):1-5. 
14. Maia H Jr, Casoy J, Correia T, Freitas LA, Pimentel K, Athayde C. The effect of oral contraceptives on aromatase expression in the eutopic endometrium of patients with endometriosis. Gynecol Endocrinol. 2008; 24(3):123-128.

15. Baskin GB, Smith SM, Marx PA. Endometrial hyperplasia, polyps and adenomyosis associated with unopposed estrogens in rhesus monkeys (Macaca mulatta). Vet Pathol. 2002;39(5):572-575.

16. Maia H Jr, Casoy J, Pimentel K, et al. Effect of oral contraceptives on vascular endothelial growth factor, Cox-2 and aromatase expression in the endometrium of uteri affected by myomas and associated pathologies. Contraception. 2008;78(6):479-485.

17. McLucas B. Does endometrial resection cause adenomyosis? J Am Assoc Gynecol Laparosc. 1994;1(4 Part 2):S21.

18. Quemere MP, Cravello L, Roger V, d'Ercole C, Blanc B. [Impact of adenomyosis on results of endometrial ablations.] Contracept Fertil Sex. 1999;27(5):357-363. French.

19. Hatok J, Zubor P, Galo S, et al. Endometrial aromatase mRNA as a possible screening tool for advanced endometriosis and adenomyosis. Gynecol Endocrinol. 2011;27(5):331-336.

20. Maia H Jr, Casoy J, Correia T, Athayde C, Valente J, Coutinho EM Activation of NF-kappaB and COX-2 expression is associated with breakthrough bleeding in patients using oral contraceptives in extended regimens. Gynecol Endocrinol. 2010;26(4):265-269.
21. Fechner S, Husen B, Thole $\mathrm{H}$, et al. Expression and regulation of estrogen-converting enzymes in ectopic human endometrial tissue. Fertil Steril. 2007;88(Suppl 4):1029-1038.

22. Yamanaka K, Xu B, Suganuma I, et al. Dienogest inhibits aromatase and cyclooxygenase-2 expression and prostaglandin E2 production in human endometriotic stromal cells in spheroid culture. Fertil Steril. 2012;97(2):477-482.

23. Maia H Jr, Haddad C, Coelho G, Casoy J. Role of inflammation and aromatase expression in the eutopic endometrium and its relationship with the development of endometriosis. Women's Health (Lond Engl). 2012;8(6):647-658.

24. Maia H Jr, Haddad C, Pinheiro N, Casoy J. Advantages of the association of resveratrol with oral contraceptives for management of endometriosis-related pain. Int J Women's Health. 2012;4:543-549.

25. Munro MG, Critchley HO, Broder MS, Fraser IS; FIGO Working Group on Menstrual Disorders. FIGO classification system (PALM-COEIN) for causes of abnormal uterine bleeding in nongravid women of reproductive age. Int J Gynaecol Obstet. 2011;113(1):3-13.
International Journal of Women's Health

\section{Publish your work in this journal}

The International Journal of Women's Health is an international, peerreviewed open-access journal publishing original research, reports, editorials, reviews and commentaries on all aspects of women's healthcare including gynecology, obstetrics, and breast cancer. The manuscript management system is completely online and includes

\section{Dovepress}

a very quick and fair peer-review system, which is all easy to use. Visit http://www.dovepress.com/testimonials.php to read real quotes from published authors.

\footnotetext{
Submit your manuscript here: http://www.dovepress.com/international-journal-of-womens-health-journal
} 